\title{
BMJ Open Prevalence of sarcopenia in older Chinese adults: a systematic review and meta-analysis
}

\author{
Caihong Xin, ${ }^{1}$ Xin Sun (D),${ }^{2,3}$ Li Lu, ${ }^{3}$ Ling Shan (D) ${ }^{3}$
}

To cite: Xin C, Sun X, Lu L, et al. Prevalence of sarcopenia in older Chinese adults: a systematic review and meta-analysis. BMJ Open 2021;11:e041879. doi:10.1136/ bmjopen-2020-041879

- Prepublication history and additional supplemental material for this paper are available online. To view these files, please visit the journal online (http://dx.doi.org/10.1136/ bmjopen-2020-041879)

CX and XS are joint first authors.

Received 22 June 2020 Accepted 21 June 2021

\section{Check for updates}

(C) Author(s) (or their employer(s)) 2021. Re-use permitted under CC BY-NC. No commercial re-use. See rights and permissions. Published by BMJ.

${ }^{1}$ Department of Endocrinology and Metabolism, Fourth People's Hospital in Shenyang, Shenyang, Liaoning, China

${ }^{2}$ Department of Endocrinology and Metabolism, First Affiliated Hospital of Soochow University, Suzhou, Jiangsu, China

${ }^{3}$ Department of Endocrinology and Metabolism, People's Hospital of Liaoning Province, Shenyang, Liaoning, China

Correspondence to

Dr Ling Shan;

shanling0113@163.com

\section{ABSTRACT}

Objective This meta-analysis was conducted to estimate the overall prevalence of sarcopenia in older Chinese adults.

Design Systematic review and meta-analysis.

Participants A literature research was conducted using the PubMed, Web of Science, China National Knowledge Infrastructure, CQVIP and Wanfang databases. The following search terms in the abstract were used: "sarcopenia" in combination with the terms "prevalence," "epidemiology" and "China." All studies published from January 2010 to November 2020 were included. The random-effect model was used to estimate the prevalence of sarcopenia. The sex-specific prevalence of sarcopenia at a $95 \% \mathrm{Cl}$ was also calculated using different criteria for defining sarcopenia.

Primary outcome measures The overall prevalence of sarcopenia in older Chinese adults.

Results In total, 23 articles were included in this metaanalysis involving 21564 participants. On the basis of the Asian Working Group for Sarcopenia criterion, the overall prevalence of sarcopenia among the elderly in China was $14 \%$ (95\% Cl $11 \%$ to $18 \%)$; the prevalence was higher in Chinese women than in men (15\% vs $14 \%)$.

Conclusions This systematic review is the first estimation of the pooled prevalence of sarcopenia in older Chinese adults. Our results suggest that China has a large number of patients with sarcopenia. These findings would be useful for sarcopenia prevention in China. There is a high degree of heterogeneity, and although there are a large number of cases and could be an emerging public health issue, more research is required to make these claims. PROSPERO registration number CRD42020223405.

\section{INTRODUCTION}

Sarcopenia is characterised by the deterioration of muscle mass and function that occurs with ageing. Several studies have shown that as age increases, skeletal muscle mass and strength are lost at a rate of $1 \%-2 \%$ annually, possibly leading to the decline of physical function and increasing the disability and mortality rates of elderly people as well as bringing serious economic and social burdens. $^{1-4}$

A practical clinical definition was reported by the European Working Group on Sarcopenia in Older People (EWGSOP) in $2010 .^{5}$

\section{Strengths and limitations of this study}

To our knowledge, this systematic review is the first estimation of the pooled prevalence of sarcopenia in Chinese older people.

- The search languages were limited to English and Chinese.

- We included limited number of high-quality studies.

- A high level of heterogeneity among included studies was observed.

Based on this EWGSOP criteria, slow walking speed $(0.8 \mathrm{~m} / \mathrm{s})$ or low grip strength $(<30 \mathrm{~kg}$ for men and $<20 \mathrm{~kg}$ for women) are indications of muscle mass decline. Later in 2011, another consensus was published by the International Working Group on Sarcopenia (IWGS) ${ }^{6}$ However, Asia is a vast region comprising different races, countries and cultures, and developing screening and diagnostic strategies for the Asian population is imperative. The Asian Working Group for Sarcopenia (AWGS) was established in 2013, and the consensus of AWGS was released based on 16 epidemiological reports on sarcopenia in six countries or regions, leading to the development of a diagnosis strategy for sarcopenia in Asia. ${ }^{7}$ The diagnostic criteria recommended by AWGS and EWGSOP are similar, and both include muscle mass, muscle strength and activity measurements. The only difference lies in the specific cut-off value of muscle strength and muscle mass.

According to statistics, the elderly population ( $\geq 60$ years old) includes $16.1 \%$ of China's total population, which is close to 250 million. In the face of a severe ageing situation, people are paying more attention to sarcopenia, but its prevalence is inconsistent across different studies. ${ }^{8-12}$ At present, there is no large-scale epidemiological survey of sarcopenia in China. The purpose of this study is to systematically determine the prevalence of sarcopenia in older Chinese adults. 


\section{METHODS}

\section{Search strategy}

We searched the PubMed, Web of Science, China National Knowledge Infrastructure (CNKI), CQVIP and Wanfang electronic databases. The search terms in the title or abstract included the following: "sarcopenia," "muscle mass" and "muscle strength" in combination with the terms "prevalence," "epidemiology" and "China." The complete search strategy is the following: PubMed: (( (sarcopenia [Title/Abstract]) OR muscle mass[Title/Abstract]) OR muscle strength[Title/ Abstract]) AND ((prevalence[Title/Abstract]) OR epidemi*[Title/Abstract]) AND ((Chinese[Title/ Abstract]) OR China[Title/Abstract]); Web of Science: ( ( TS=sarcopenia) OR TS=muscle mass $)$ OR TS=muscle strength) AND ((TS=prevalence) OR TS=epidemi*) AND ((TS=Chinese) OR TS=China); CNKI: $(\mathrm{AB}=$ sarcopenia $\mathrm{OR} A B=$ muscle mass $\mathrm{OR} A B=$ muscle strength $) \mathrm{AND}$ $(\mathrm{AB}=$ prevalence $\mathrm{OR} \mathrm{AB}=$ epidemiology); $\mathrm{CQVIP}:(\mathrm{R}=$ sarcopenia $+\mathrm{R}=$ muscle mass $+\mathrm{R}=$ muscle strength $) *(\mathrm{R}=$ prevalence $+\mathrm{R}=$ epidemiology); and Wanfang: Abstract: (sarcopenia +muscle mass+muscle strength) * (prevalence +epidemiology). All studies published from January 2010 to November 2020 were included. The references of the retrieved articles were examined to identify additional eligible studies, and unpublished studies were excluded. The search languages were limited to English and Chinese. This systematic review and meta-analysis was registered in PROSPERO. This meta-analysis was conducted to estimate the overall prevalence of sarcopenia in older Chinese adults.

\section{Patient and public involvement}

No patients were involved in this study.

\section{Inclusion and exclusion criteria}

Inclusion of the selected studies was based on the following criteria: (1) a cross-sectional study or retrospective study, (2) a sample size of $\geq 100$ elderly Chinese participants (age $\geq 60$ years), (3) sufficient information on sarcopenia at the corresponding $95 \% \mathrm{CI}$ and $p$ value and (4) the definition of sarcopenia in line with the AWGS, EWGSOP or IWGS standards.

Exclusion of the selected studies was based on the following criteria: (1) same data from different studies, (2) studies defining sarcopenia only as reduction of muscle mass or strength and (3) the subjects include a specific population, such as patients living in a nursing home or those with disability. Reviews, case reports, letters and animal researches were also excluded.

\section{Data extraction and methodological quality assessment}

Information from the selected studies was extracted by two independent researchers, including the first author's name, publication year, sample, diagnostic criteria, participants, setting and prevalence. Two independent reviewers who were not blinded to the authors or journals assessed the risk of bias in the included studies using the Newcastle-Ottawa Scale (NOS). ${ }^{13}$ The NOS is

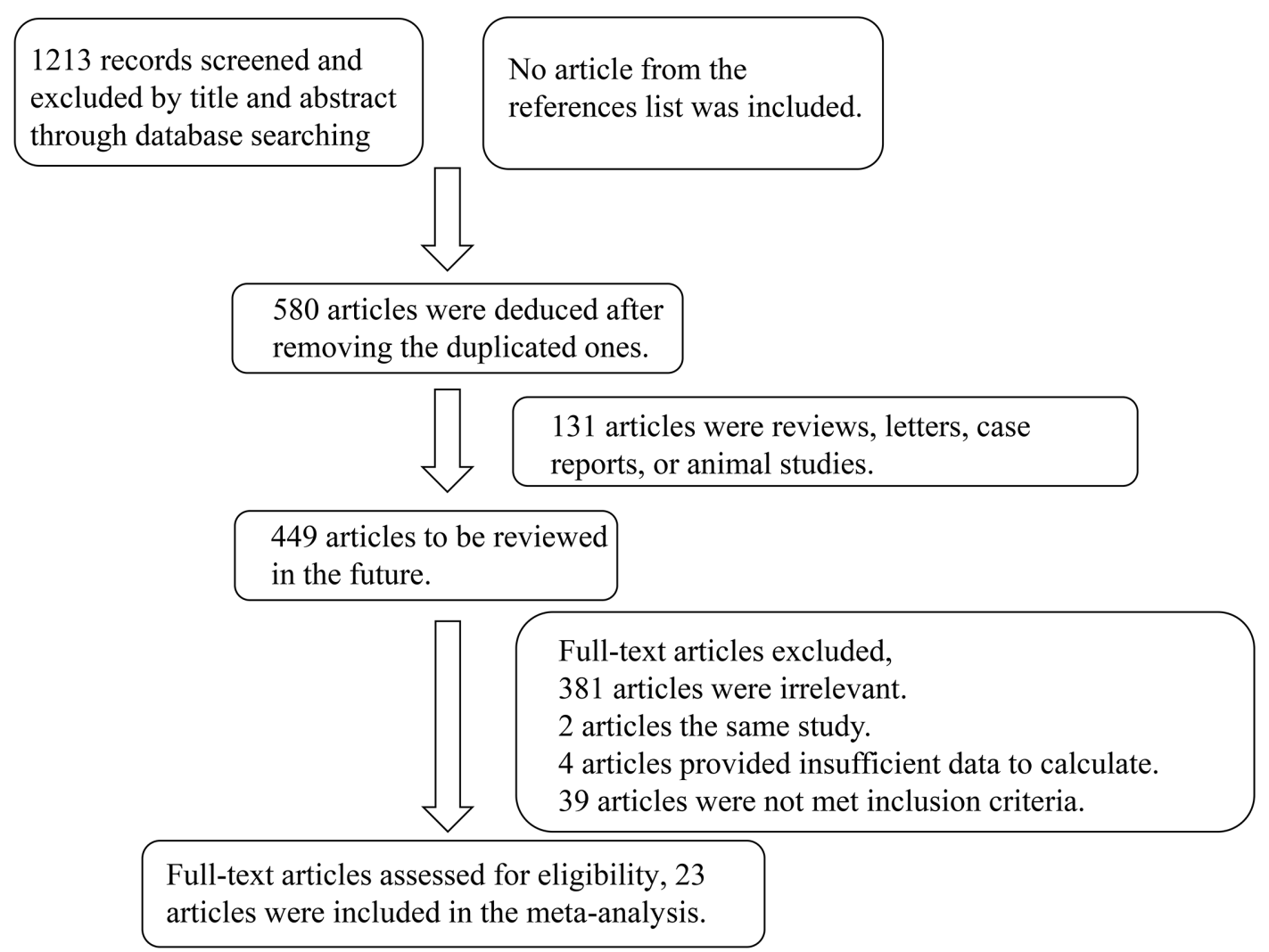

Figure 1 Flowchart of the detailed procedure for the inclusion or exclusion of selected studies. 


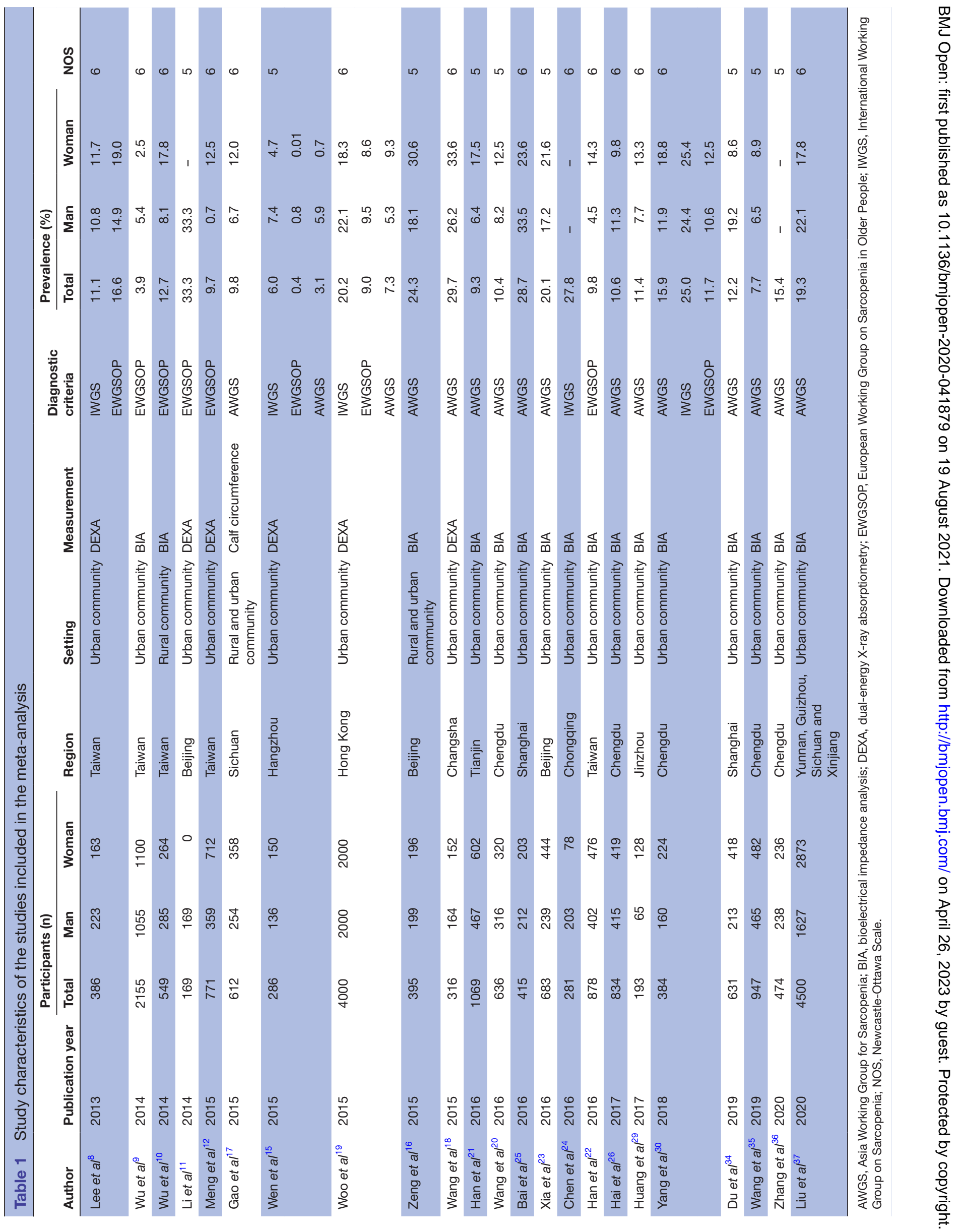




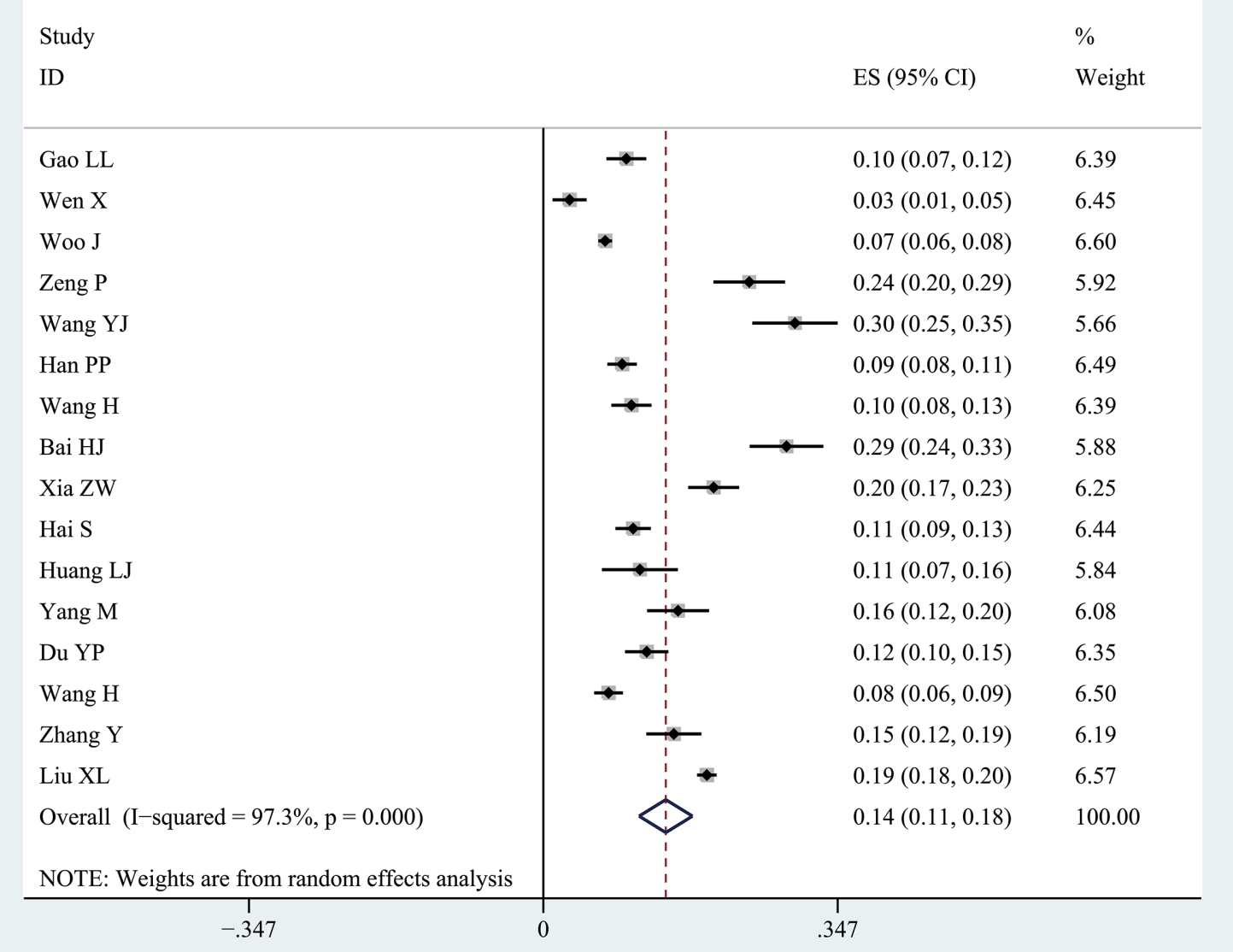

Figure 2 Forest plots for the total prevalence of sarcopenia among the elderly Chinese population according to the Asian Working Group for Sarcopenia standard. Diamond represents the pooled ES at 95\% Cl. ES, effect size.

recommended by the Cochrane Handbook for Systematic Reviews of Interventions. ${ }^{14}$ Each included study was analysed in terms of three domains, namely, representativeness of the study group selection, group comparability and exposure or outcome ascertainment, using the "star system". NOS scores ranged from zero to nine stars. The two researchers independently assessed the studies by discussion, comparison of findings and resolution of differences through consensus formation. If no consensus was reached, a third researcher was commissioned to resolve the difference.

\section{Statistical analysis}

We used a systematic analysis approach to calculate the pooled prevalence of sarcopenia for all eligible studies, and heterogeneity among these studies was assessed using Cochran's $Q$ test and I $^{2}$ Statistical Index. The randomeffect model was used to combine the prevalence of sarcopenia. We additionally performed sensitivity analysis to evaluate the influence of any given study on the pooled estimate. Publication bias was evaluated using Egger's test. Subgroup analyses were also performed. The significance level was set at $\mathrm{p}<0.05$. All statistical analyses were performed using Stata V.12.0.

\section{RESULTS}

\section{Study characteristics}

In total, 1213 studies were retrieved from the databases, and 580 articles remained after the exclusion of duplicates. After screening for eligibility based on the full text, a total of 23 articles were included as per the criteria shown in figure $1 .^{8-12 ~ 15-37}$ Altogether, the 23 articles included 21564 participants in China between 2010 and 2020. The selected studies' characteristics are summarised in table 1. On the basis of the NOS, the score of selected studies was all above five stars; therefore, all articles were included.

\section{Results of the meta-analysis}

According to the AWGS standard, the total prevalence of sarcopenia among Chinese elderly people was $14 \%$ (95\% CI $11 \%$ to $18 \%$, figure 2), and total prevalence rates of sarcopenia among the elderly in China based on the IWGS and EWGSOP standards were 18\% (95\% CI $11 \%$ to $25 \%$, figure 3 ) and $10 \%$ (95\% CI $7 \%$ to $14 \%$, figure 4 ), respectively.

Subgroup analyses were performed to determine the influence of sex on the prevalence of sarcopenia. Sarcopenia prevalence among elderly women was higher than that in men as per the AWGS and EWGSOP standards 
Study

ID

ES $(95 \% \mathrm{CI})$

$\%$

Weight

Lee WJ

Wen X

Woo J

Chen Z

Yang M

Overall $(\mathrm{I}-$ squared $=96.9 \%, \mathrm{p}=0.000)$

NOTE: Weights are from random effects analysis

Figure 3 Forest plots for the total prevalence of sarcopenia among the elderly Chinese population according to the International Working Group on Sarcopenia standard. Diamond represents the pooled ES at 95\% CI. ES, effect size.

\begin{tabular}{|c|c|c|c|}
\hline \multicolumn{3}{|l|}{ Study } & \multirow{2}{*}{$\begin{array}{l}\% \\
\text { Weight }\end{array}$} \\
\hline ID & & ES $(95 \% \mathrm{CI})$ & \\
\hline Lee WJ & $\rightarrow$ & $0.17(0.13,0.20)$ & 10.94 \\
\hline Wu IC & - & $0.04(0.03,0.05)$ & 12.44 \\
\hline $\mathrm{Wu} \mathrm{CH}$ & $\circ$ & $0.13(0.10,0.15)$ & 11.58 \\
\hline Li M & & $0.33(0.21,0.46)$ & 4.82 \\
\hline Meng NH & $\rightarrow$ & $0.10(0.08,0.12)$ & 11.98 \\
\hline Wen $\mathrm{X}$ & 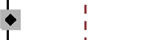 & $0.00(-0.00,0.01)$ & 12.47 \\
\hline Woo J & 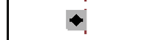 & $0.09(0.08,0.10)$ & 12.43 \\
\hline Han DS & $\rightarrow$ & $0.10(0.08,0.12)$ & 12.04 \\
\hline Yang M & & $0.12(0.08,0.15)$ & 11.30 \\
\hline Overall $(\mathrm{I}-\mathrm{squared}=98.0 \%, \mathrm{p}=0.000)$ & & $0.10(0.07,0.14)$ & 100.00 \\
\hline NOTE: Weights are from random effects analysis & & & \\
\hline $\begin{array}{ll} \\
-.456\end{array}$ & 0 & & \\
\hline
\end{tabular}

Figure 4 Forest plots for the total prevalence of sarcopenia among the elderly Chinese population according to the European Working Group on Sarcopenia in Older People standard. Diamond represents the pooled ES at $95 \% \mathrm{Cl}$. ES, effect size. 


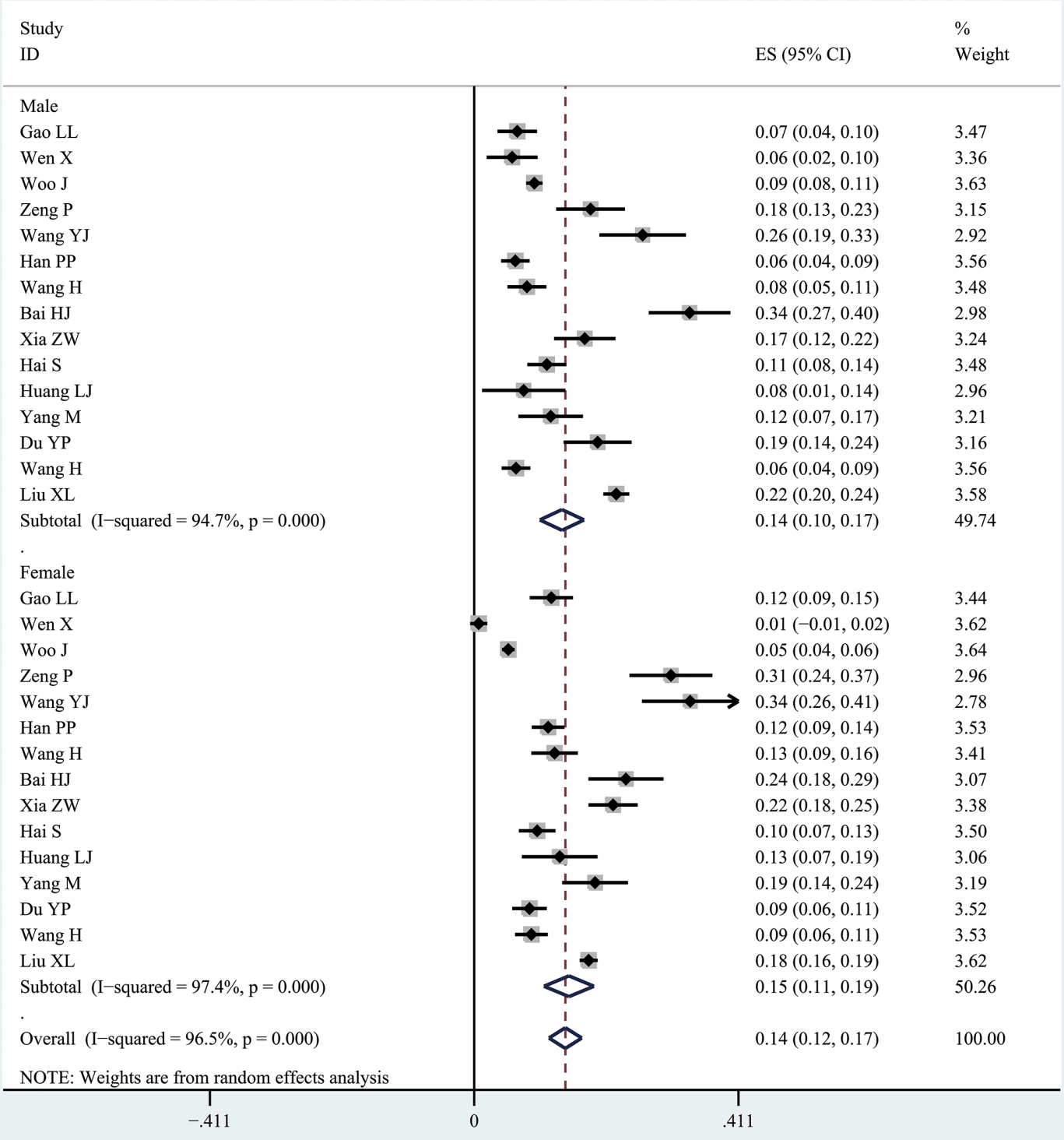

Figure 5 Forest plots for the prevalence of sarcopenia among male and female elderly people according to the Asian Working Group for Sarcopenia standard. Diamond represents the pooled ES at $95 \% \mathrm{CI}$. ES, effect size.

(figures 5-7). Table 2 shows the subgroup analyses results based on the different diagnostic criteria. The prevalence of sarcopenia estimated using the bioelectrical impedance analysis (BIA) method was higher than that estimated using the dual energy X-ray absorptiometry (DEXA) method as per the AWGS and IWGS standards.

\section{Sensitivity analysis and publication bias}

Sensitivity analysis was performed to examine the influence of each study. We found no significant difference between the results of the sensitivity analysis and our previous estimates, indicating that our statistical results were relatively credible (online supplemental figures S1S3). The articles obtained from the databases were carefully and comprehensively searched. Additionally, Egger's test was conducted to determine whether potential publication bias existed in the reviewed literature. The results
( $>0.05)$ suggested that there was no publication bias (online supplemental figure S4-S6).

\section{DISCUSSION}

To the best of our knowledge, this study is the first metaanalysis of the prevalence of sarcopenia in older Chinese adults. Sarcopenia is an age-related disease characterised by progressive deterioration in skeletal muscle fibre quality, strength and function. Currently, sarcopenia has been internationally recognised as a new type of geriatric syndrome and has attracted worldwide attention. In 2017, Shafiee et al conducted a meta-analysis on the prevalence of sarcopenia. Their results showed that sarcopenia prevalence among the elderly in communities worldwide was approximately $10 \%$; this was higher among non-Asian 


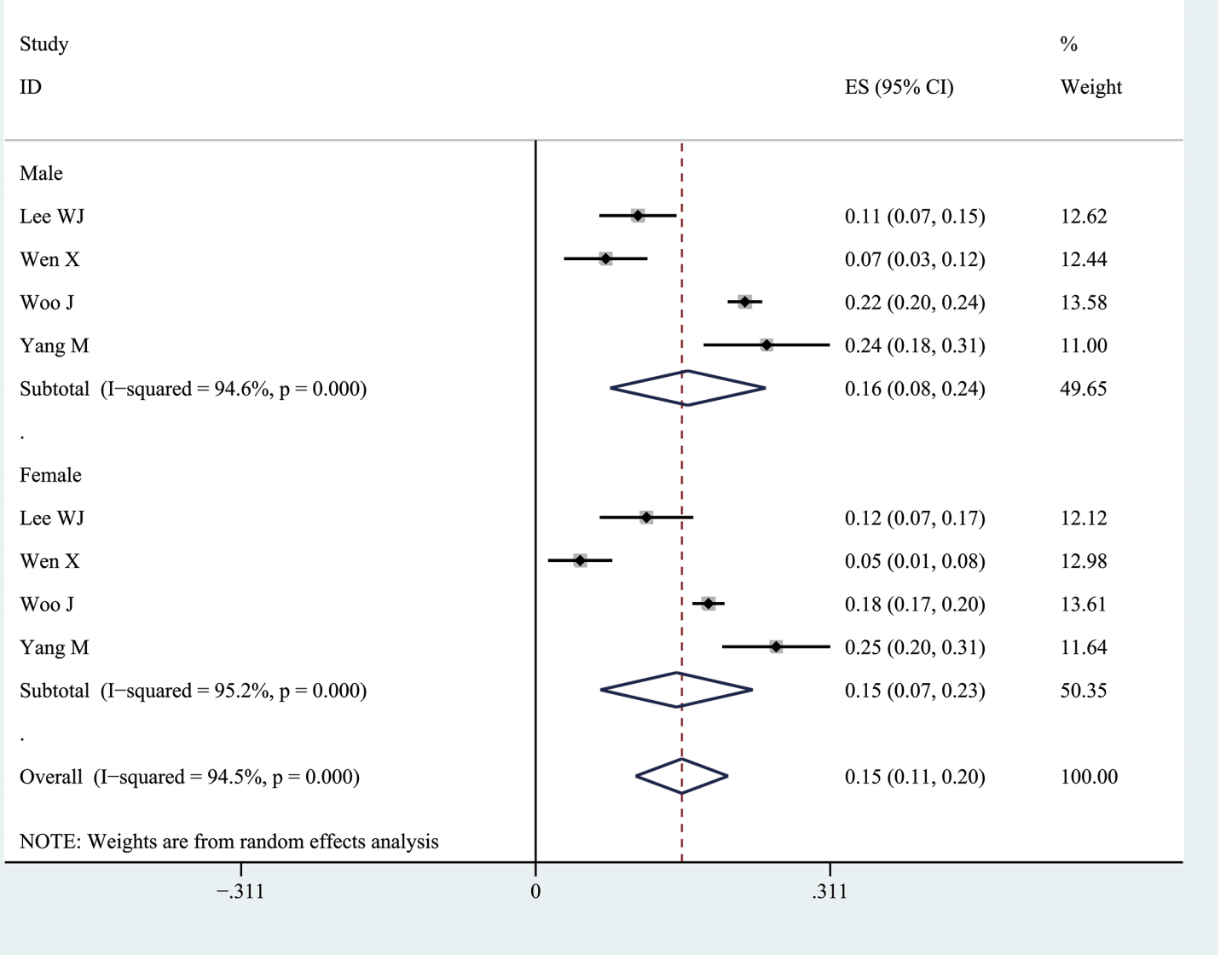

Figure 6 Forest plots for the prevalence of sarcopenia among male and female elderly people according to the International Working Group on Sarcopenia standard. Diamond represents the pooled ES at 95\% Cl. ES, effect size.

individuals than Asians in both sexes. ${ }^{38}$ A similar study was performed in Japan, where sarcopenia prevalence among the elderly Japanese was $9.9 \%$ (95\% CI $6.2 \%$ to $15.4 \%$ ) according to the AWGS standard. ${ }^{39}$ The results of our study showed that the prevalence of sarcopenia was $14 \%$ (95\% CI $11 \%$ to $18 \%$ ) in older Chinese adults, which was much higher than the results of the aforementioned studies. ${ }^{40}{ }^{41}$ This high prevalence may be related to the Chinese diet structure. Although protein is the raw material for muscle synthesis, Chinese people, particularly the older population, have higher carbohydrate intake and less protein intake. ${ }^{42}$ Therefore, more attention should be given to the prevention and control of sarcopenia in older Chinese adults.

Here, we found that the prevalence of sarcopenia among women was higher than that in men, which was consistent with other meta-analyses. ${ }^{38-40}$ This is mainly because men eat and exercise more than women; this supports muscle synthesis. Most of the studies included in our meta-analysis used the BIA method because of its affordability and portable equipment. BIA is a widely used non-invasive method for measuring human body composition. The principle behind this method is to record the different electrical resistances of different tissues using electrodes placed on the body surface and then to measure muscle mass using an image reconstruction method. DEXA is also commonly used to evaluate muscle mass. DEXA can accurately distinguish the whole body and local muscles as well as the fats and bones. However, the limited number of studies using DEXA included in this meta-analysis may influence our results. As is known, ageing increases the prevalence of sarcopenia; however, due to the different age classifications in the included studies, we cannot obtain any conclusion.

The meta-analysis results show that the heterogeneity is high, which may be related to factors such as age, race and living environment. In addition, there are differences in the prevalence of sarcopenia in rural and urban areas. China is a country with a vast territory and multiethnic people. The findings of this meta-analysis should be interpreted with caution.

First, the limitation of the present study is that the main data are from urban populations and that studies on the prevalence of sarcopenia in Chinese rural areas are rare; this impacts the calculation of the total sarcopenia prevalence. Second, the prevalence of sarcopenia in people aged $60-70$ years and $70-80$ years might be different. Not all studies presented their data according to age group, so we cannot accurately estimate the prevalence in terms of age subgroup. Third, most of the studies were performed in large cities or economically developed areas. All of 


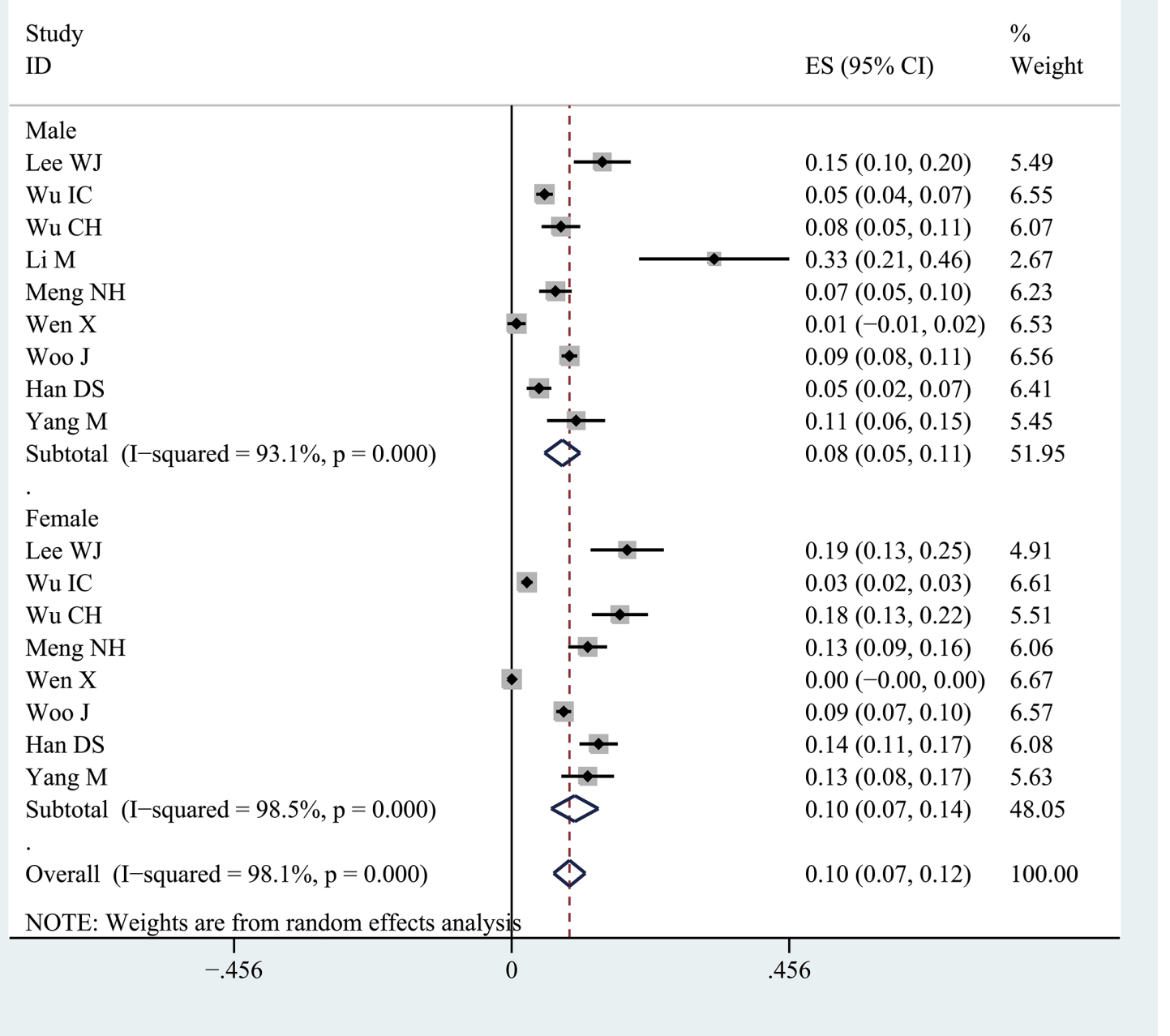

Figure 7 Forest plots for the prevalence of sarcopenia among male and female elderly people according to the European Working Group on Sarcopenia in Older People standard. Diamond represents the pooled ES at $95 \% \mathrm{CI}$. ES, effect size.

these factors affected our results, and as such, they should be interpreted cautiously as further research is needed.

\section{CONCLUSIONS}

To the best of our knowledge, the present systematic review is the first to estimate the pooled prevalence of sarcopenia in older Chinese adults. The results of the present systematic review reveal that China has a

\begin{tabular}{|c|c|c|}
\hline & DEXA & BIA \\
\hline AWGS & $13(5-21)$ & $15(12-19)$ \\
\hline IWGS & $12(3-22)$ & $26(23-29)$ \\
\hline EWGSOP & $12(6-18)$ & $9(5-14)$ \\
\hline
\end{tabular}

AWGS, Asia Working Group for Sarcopenia; BIA, bioelectrical impedance analysis; DEXA, dual-energy X-ray absorptiometry; EWGSOP, European Working Group on Sarcopenia in Older People; IWGS, International Working Group on Sarcopenia. large number of sarcopenia cases and sarcopenia is an emerging public health issue in China. There is a high degree of heterogeneity, and although there are a large number of cases and could be an emerging public health issue, more research is required to make these claims.

Acknowledgements We thank Enago for English-language editing.

Contributors LS designed the study. XS and LL searched databases and collected the data. CX and LS assessed the quality of the study. LS performed the analysis. XS and CX wrote the manuscript. XS modified the manuscript in the revision. All authors contributed to this systematic review and meta-analysis.

Funding This research was financially supported by the National Natural Science Foundation of China (grant No. 81800697).

Competing interests None declared.

Patient consent for publication Not required.

Ethics approval This study did not require ethical approval as the data used have been published previously and hence are already in the public domain.

Provenance and peer review Not commissioned; externally peer reviewed.

Data availability statement All data relevant to the study are included in the article or uploaded as supplemental information.

Supplemental material This content has been supplied by the author(s). It has not been vetted by BMJ Publishing Group Limited (BMJ) and may not have been 
peer-reviewed. Any opinions or recommendations discussed are solely those of the author(s) and are not endorsed by BMJ. BMJ disclaims all liability and responsibility arising from any reliance placed on the content. Where the content includes any translated material, BMJ does not warrant the accuracy and reliability of the translations (including but not limited to local regulations, clinical guidelines, terminology, drug names and drug dosages), and is not responsible for any error and/or omissions arising from translation and adaptation or otherwise.

Open access This is an open access article distributed in accordance with the Creative Commons Attribution Non Commercial (CC BY-NC 4.0) license, which permits others to distribute, remix, adapt, build upon this work non-commercially, and license their derivative works on different terms, provided the original work is properly cited, appropriate credit is given, any changes made indicated, and the use is non-commercial. See: http://creativecommons.org/licenses/by-nc/4.0/.

\section{ORCID iDs}

Xin Sun http://orcid.org/0000-0003-1409-4661

Ling Shan http://orcid.org/0000-0003-2019-9718

\section{REFERENCES}

1 Cederholm T, Morley JE. Sarcopenia: the new definitions. Curr Opin Clin Nutr Metab Care 2015;18:1-4.

2 Frontera WR, Hughes VA, Fielding RA, et al. Aging of skeletal muscle: a 12-yr longitudinal study. J App/ Physiol 2000;88:1321-6.

3 Delmonico MJ, Harris TB, Lee J-S, et al. Alternative definitions of sarcopenia, lower extremity performance, and functional impairment with aging in older men and women. J Am Geriatr Soc 2007;55:769-74.

4 Woo J. Sarcopenia. Clin Geriatr Med 2017;33:305-14.

5 Cruz-Jentoft AJ, Baeyens JP, Bauer JM, et al. Sarcopenia: european consensus on definition and diagnosis: report of the european working group on sarcopenia in older people. Age Ageing 2010;39:412-23.

6 Fielding RA, Vellas B, Evans WJ, et al. Sarcopenia: an undiagnosed condition in older adults. Current consensus definition: prevalence, etiology, and consequences. International Working group on sarcopenia. J Am Med Dir Assoc 2011;12:249-56.

7 Chen L-K, Liu L-K, Woo J, et al. Sarcopenia in Asia: consensus report of the asian working group for sarcopenia. J Am Med Dir Assoc 2014;15:95-101.

8 Lee W-J, Liu L-K, Peng L-N, et al. Comparisons of sarcopenia defined by IWGS and EWGSOP criteria among older people: results from the I-Lan longitudinal aging study. J Am Med Dir Assoc 2013;14:528.e1-528.e7.

9 Wu I-C, Lin C-C, Hsiung CA, et al. Epidemiology of sarcopenia among community-dwelling older adults in Taiwan: a pooled analysis for a broader adoption of sarcopenia assessments. Geriatr Gerontol Int 2014;14 Suppl 1:52-60.

10 Wu C-H, Chen K-T, Hou M-T, et al. Prevalence and associated factors of sarcopenia and severe sarcopenia in older taiwanese living in rural community: the tianliao old people study 04. Geriatr Gerontol Int 2014;14 Suppl 1:69-75.

$11 \mathrm{Li} \mathrm{M}, \mathrm{Hu}$ Y, Dong HY. Compare different measurement for prevalence of sarcopenia in a cohort of healthy community dwellingely in Beijing area. Chin. J. Health Care Med 2014;16:426-9.

12 Meng N-H, Li C-I, Liu C-S, et al. Comparison of height- and weightadjusted sarcopenia in a Taiwanese metropolitan older population. Geriatr Gerontol Int 2015;15:45-53.

13 Wells GA, Shea B, O'Connell D. The newcastle-ottawa scale (NOS) for assessing the quality of nonrandomised studies in meta-analyses. Available: http://www.ohri.ca/programs/clinical_epidemiology/oxford. asp [Accessed 05 Aug 2014].

14 Higgins JPT, Green S. Cochrane handbook for systematic reviews of interventions version 5.1.0, 2011. http://www.cochrane-handbook. org

15 Wen X, An P, Chen WC, et al. Comparisons of sarcopenia prevalence based on different diagnostic criteria in Chinese older adults. J Nutr Health Aging 2015;19:342-7.

16 Zeng P, Wu S, Han Y, et al. Differences in body composition and physical functions associated with sarcopenia in Chinese elderly: reference values and prevalence. Arch Gerontol Geriatr 2015;60:118-23.

17 Gao L, Jiang J, Yang M, et al. Prevalence of sarcopenia and associated factors in Chinese community-dwelling elderly: comparison between rural and urban areas. J Am Med Dir Assoc 2015;16:1003.e1-1003.e6.
18 Wang Y-J, Wang Y, Zhan J-K, et al. Sarco-osteoporosis: prevalence and association with frailty in Chinese community-dwelling older adults. Int J Endocrinol 2015;2015:1-8.

19 Woo J, Leung J, Morley JE. Defining sarcopenia in terms of incident adverse outcomes. J Am Med Dir Assoc 2015;16:247-52.

20 Wang $\mathrm{H}$, Hai S, Cao L, et al. Estimation of prevalence of sarcopenia by using a new bioelectrical impedance analysis in Chinese community-dwelling elderly people. BMC Geriatr 2016;16:216.

21 Han P, Kang L, Guo Q, et al. Prevalence and factors associated with sarcopenia in Suburb-dwelling older Chinese using the Asian Working group for sarcopenia definition. J Gerontol A Biol Sci Med Sci 2016;71:529-35.

22 Han D-S, Chang K-V, Li C-M, et al. Skeletal muscle mass adjusted by height correlated better with muscular functions than that adjusted by body weight in defining sarcopenia. Sci Rep 2016;6:19457.

23 Xia Z, Meng L, Man Q, et al. [Analysis of the dietary factors on sarcopenia in elderly in Beijing]. Wei Sheng Yan Jiu 2016;45:388-93.

24 Chen Z, Dai R, Gu XH. Prevalence of sarcopenia and quality of life in retired cadres of a military unit. Chin. J. Osteopo. Rosis. \& Bone Miner. Res 2016;9:264-8.

25 Bai H-J, Sun J-Q, Chen M, et al. Age-related decline in skeletal muscle mass and function among elderly men and women in Shanghai, China: a cross sectional study. Asia Pac J Clin Nutr 2016;25:326-32

26 Hai S, Wang H, Cao L, et al. Association between sarcopenia with lifestyle and family function among community-dwelling Chinese aged 60 years and older. BMC Geriatr 2017;17:187.

27 Ma Y, Bian AL, Wang XF. Difference of the prevalence rate of sarcopenia in elderly people with different gender and age in Tianjin. Chin. General Practice 2017;20:3267-71.

28 Wu ZN, Wang YB, Shu FQ. Epidemiological investigation of sarcopenia in the nursing homes in Fengxian district and analysis of their risk factors. Shanghai Med 2017;38:37-40.

29 Huang LJ, Liu YC, Wen YF. State and associated factors of sarcopenia in Jinzhou community elderly people. Modern Preve. Med 2017;44:4458-60.

30 Yang M, Hu X, Xie L, et al. Screening sarcopenia in communitydwelling older adults: SARC-F vs SARC-F combined with calf circumference (SARC-CalF). J Am Med Dir Assoc 2018;19:277. e1-277.e8.

31 Zeng Y, Hu X, Xie L, et al. The prevalence of sarcopenia in Chinese elderly nursing home residents: a comparison of 4 diagnostic criteria. J Am Med Dir Assoc 2018;19:690-5.

32 Wang YN, Xu JL, Song HL. Influencing factors of sarcopenia among elderly patients. Modern Clinical Nursing 2018;17:45-50.

33 Yang L-J, Wu G-H, Yang Y-L, et al. Nutrition, physical exercise, and the prevalence of sarcopenia in elderly residents in nursing homes in China. Med Sci Monit 2019;25:4390-9.

$34 \mathrm{Du} \mathrm{Y}$, Wang $\mathrm{X}$, Xie $\mathrm{H}$, et al. Sex differences in the prevalence and adverse outcomes of sarcopenia and sarcopenic obesity in community dwelling elderly in East China using the AWGS criteria. BMC Endocr Disord 2019;19:109.

35 Wang H, Hai S, Liu Y, et al. [Prevalence of sarcopenia and associated factors in community-dwelling elderly populations in chengdu china]. Sichuan Da Xue Xue Bao Yi Xue Ban 2019;50:224-8.

36 Zhang Y, Chen X, Hou L, et al. Prevalence and risk factors governing the loss of muscle function in elderly sarcopenia patients: a longitudinal study in China with 4 years of follow-up. J Nutr Health Aging 2020;24:518-24.

37 Liu X, Hao Q, Yue J, et al. Sarcopenia, obesity and sarcopenia obesity in comparison: prevalence, metabolic profile, and key differences: results from WCHAT study. J Nutr Health Aging 2020;24:429-37.

38 Shafiee G, Keshtkar A, Soltani A, et al. Prevalence of sarcopenia in the world: a systematic review and meta- analysis of general population studies. J Diabetes Metab Disord 2017;16:21.

39 Makizako H, Nakai Y, Tomioka K, et al. Prevalence of sarcopenia defined using the Asia working group for sarcopenia criteria in japanese community-dwelling older adults: a systematic review and meta-analysis. Phys Ther Res 2019;22:53-7.

40 Diz JBM, Leopoldino AAO, Moreira BdeS, et al. Prevalence of sarcopenia in older brazilians: a systematic review and metaanalysis. Geriatr Gerontol Int 2017;17:5-16.

41 Papadopoulou SK, Tsintavis P, Potsaki P, et al. Differences in the prevalence of sarcopenia in community-dwelling, nursing home and hospitalized individuals. A systematic review and meta-analysis. $J$ Nutr Health Aging 2020;24:83-90.

42 Han A, Sun T, Ming J, et al. Are the Chinese moving toward a healthy diet? Evidence from macro data from 1961 to 2017. Int J Environ Res Public Health 2020;17:5294. 